\title{
The role of mobile technology for resident assessment of surgical skills in the CBME era
}

\author{
Kashif Visram, $M D$ \\ Queen's University, Kingston, ON, Canada
}

Cite as: Can Urol Assoc J 2019;13(2):51-2. hitp://dx.doi.org/10.5489/cuai.5849

See related article on page 45

T he transition to competency-based medical education (CBME) is in full swing for Canadian urology, as all new cohorts of residents will be trained under this model. In the past, competence was assumed to be achieved after completion of a five-year time-based residency. Now, trainees are expected to demonstrate competence, through frequent assessment, in clearly defined entrustable professional activities (EPAs). Once a trainee achieves competence in all EPAs, they demonstrate they have the capability to practice urology independently.

Technical skill proficiency is an integral component of independent urological practice, yet how trainees obtains it over the course of their residency is not well understood. ${ }^{1}$ Certainly, direct observation of junior trainees by more experienced colleagues providing well thought out feedback contributes to this process. Fitzpatrick et al have correctly identified the need for a user-friendly platform that can objectively track resident surgical skill development in the CBME era. ${ }^{2}$ Their model amalgamates the surgical case log, a commonly used tool that predates $\mathrm{CBME}$, with regular selective triggering of structured assessment from within the log itself to measure resident progress. Furthermore, they implement this tool using an online platform with the aim to enhance uptake and ease of use for both residents and staff.

Efficient use of technology will continue to be essential in facilitating the increased number of evaluations required for CBME residents, hence the development of online and mobile applications for this purpose should be applauded. In this study, residents and faculty, although the latter somewhat less so $(100 \%$ vs. $67 \%)$, reported being comfortable with using mobile technology, which is unsurprising given the ubiquitous nature of smartphones and tablets. Building familiarity with the mobile application itself, like anything new, requires time and available appropriate training. Ensuring that adequate technical support and good wireless coverage is available to residents is necessary to allow for proper use. Integrating the surgical case log with individual surgical performance evaluations creates a dynamic tool for overall assessment. Throughout one's residency, you could view the volume of surgery that one was involved with, alongside correlated information elucidating the development of proficiencies. However, to be valuable it requires buy-in from residents and staff — the surgical log must be complete and evaluation must be frequent and consistent all through residency.

Integrated systematic controls could help circumvent this issue. In their model, Fitzpatrick et al built in program director-driven selection of cases for evaluation, as well as 24-hour turnaround time for evaluations to be completed in order to keep them relevant and accurate. Taking the onus off the resident and staff involved in the evaluation minimizes selection bias. Residents and staff cannot pick certain cases and evaluators are predetermined. However, this places a lot of responsibility on program directors, who are likely overseeing many residents, to ensure that frequent evaluation is being triggered and that appropriate skills are being evaluated, which would be burdensome. To improve on this, academic mentors or staff assigned to individual residents could be responsible for overseeing the assessment process.

The value of resident-driven assessment should not be understated. Residents should be able to obtain objective assessment for skills they have identified as things they are trying to improve on to drive self-directed learning. This would also be important for residents requiring evaluation in certain areas to record proficiency for a given EPA. Additionally, allowing staff to trigger assessments on residents when they feel there are valuable teaching points they wish to convey also enhances resident learning. A blended model likely provides the optimal assessment strategy.

Ensuring timely feedback also poses an issue. With a 24-hour expiry on assessment, you may increase the relevance of the assessment but potentially at the cost of obtaining an assessment in the first place. A more in-depth analysis looking at the rate of expired evaluations weighed against the importance of timely feedback needs to be done to ascertain how best to optimize this. 
Verbal feedback in the operating room is an invaluable learning experience that must not be forgotten in the CBME era, where more frequent formal assessment will be expected of staff. As seen in this study, residents felt that verbal feedback remained either unchanged or increased, which is reassuring. As the demand for more formalized assessments increases, it is important to ensure that verbal feedback and bedside teaching does not decline as a result.

This study highlights the utility of technology for enhancing assessment of surgical residents. It demonstrates that highquality, frequent assessments can be easily integrated into surgical training programs, which will be required as we progress with CBME. With tinkering and adjustment to how the assessments are delivered, processed, and completed, it can be a valuable tool for developing the urologists of tomorrow.
Competing interests: The author reports no competing personal or financial interest related to this work.

\section{References}

1. Fahim C, Wagner N, Nousiainen MT, et al. Assessment of technical skills competence in the operating room: A systematic and scoping review. Acad Med 2018;93:794-808. https://doi.org/10.1097/ACM.0000000000001902

2. Fitzpatrick R, Paterson NR, Watterson J, et al. Development and implementation of a mobile version of the 0-SCORE assessment tool and case log for competency-based assessment in urology residency training: An initial assessment of utilization and acceptance amongst residents and faculty Can Urol Assoc J 2018 July 31; Epub ahead of print. http://dx.doi.org/10.5489/cuai.5482

Correspondence: Dr. Kashif Visram, Queen's University, Kingston, 0N, Canada; kvisram@qmed.ca 